\title{
A Web-based family problem-solving intervention for families of children with traumatic brain injury
}

\author{
SHARI L. WADE \\ Cincinnati Children's Hospital Medical Center \\ and College of Medicine, University of Cincinnati, Cincinnati, Ohio \\ CHRISTOPHER R. WOLFE \\ Miami University, Oxford, Ohio \\ and \\ JOHN P. PESTIAN \\ Cincinnati Children's Hospital Medical Center \\ and College of Medicine, University of Cincinnati, Cincinnati, Ohio
}

\begin{abstract}
We developed a Web-based intervention for pediatric traumatic brain injury (TBI) and examined its feasibility for participants with limited computer experience. Six families, including parents, siblings, and children with TBI, were given computers, Web cameras, and high-speed Internet access. Weekly videoconferences with the therapist were conducted after participants completed on-line interactive experiences on problem solving, communication, and TBI-specific behavior management. Families were assigned to videoconference with NetMeeting (iBOT cameras) or ViaVideo. Participants ranked the Web site and videoconferences as moderately to very easy to use. ViaVideo participants rated videoconferencing significantly more favorably relative to face-to-face meetings than did NetMeeting participants. Both the Web site and videoconferencing were rated as very helpful. All families demonstrated improved outcomes on one or more target behaviors, including increased understanding of the injury and improved parent-child relationships. All parents and siblings and all but 1 child with TBI said they would recommend the program to others. We conclude that a face-to-face intervention can be successfully adapted to the Web for families with varied computer experience.
\end{abstract}

Traumatic brain injury (TBI) creates significant stress for both child and family (Brooks, 1991; Brown, Chadwick, Shaffer, Rutter, \& Traub, 1981; Rivara et al., 1992; Wade, Taylor, Drotar, Stancin, \& Yeates, 1998). The majority of children with moderate to severe brain injuries experience some cognitive and behavioral impairment (Taylor et al., 2002; Yeates et al., 2002). Longer term follow-up studies indicate that emerging behavior problems represent the most persistent sequelae of TBI in children (Fay et al., 1994; Fletcher, Ewing-Cobbs, Miner, Levin, \& Eisenberg, 1990; Max, Koele, et al., 1998; Rutter, 1981; Schwartz et al., 2003). These findings highlight the importance of social and behavioral outcomes following TBI.

This work was supported by Grant HD40942-02, NCMRR, National Institutes of Health. We wish to acknowledge the contributions of Arthur Nezu, Christine Nezu, and Mark Ylvisaker in developing the intervention content. We also wish to acknowledge the assistance of Tanya Maines Brown in developing the Web site and Sarah Holterman in data collection and coding. These results were presented in part at the 2003 annual conference of the Society for Computers in Psychology in Vancouver, BC. Correspondence should be addressed to S. L. Wade, Division of Pediatric Physical Medicine and Rehabilitation, Cincinnati Children's Hospital Medical Center, 3333 Burnet Avenue, Cincinnati, OH 45229-3039 (e-mail: shari.wade@cchmc.org).
Following acute hospitalization, families report needing support as well as additional information about the child's injury and possible medical, cognitive, and behavioral sequelae, as well as the availability of financial and medical resources (Hawley, Ward, Magnay, \& Long, 2002; M. Marks, Sliwinski, \& Gordon, 1993; Wade, Taylor, Drotar, Stancin, \& Yeates, 1996). Other studies have documented a high level of psychological symptoms and distress in family members following TBI (Rivara et al., 1992; Rivara et al., 1996; Wade et al., 1998). Prospective investigations of families following pediatric TBI have reported that this distress can be felt as long as 3 years following the injury (Rivara et al., 1996; Wade et al., 2002). These findings suggest that pediatric TBI has a profound and persistent negative impact on both the child and the family that is not typically addressed by existing services.

Four prospective studies to date have demonstrated a relationship between the family environment and cognitive and behavioral outcomes among school-aged children with TBI (Kinsella, Ong, Murtaugh, Prior, \& Sawyer, 1999; Max, Castillo, et al., 1998, Taylor et al., 1999; Yeates et al., 1997). These studies suggest that child recovery and family adaptation exert reciprocal influences upon one another over time. Thus, family-centered inter- 
ventions provide a viable means for improving both child and family outcomes.

\section{Family Interventions Following Pediatric TBI}

Our review of the available literature yielded only two investigations to date that have evaluated interventions for families following pediatric TBI. Singer and colleagues examined reductions in parental anxiety and depression in parents of children with TBI following either a stress management program or an informational support group (Singer et al., 1994). Parents in the stress management group experienced significantly greater reductions in anxiety and depression than did those receiving the informational support intervention, suggesting that education alone is not sufficient to reduce symptoms. Despite a small sample size, the study highlights the potential utility of family/caregiver interventions following pediatric TBI. Wade, Michaud, and Brown (2004) developed and tested a family-centered intervention in a randomized trial with 32 families of children with moderate to severe TBI. The intervention consisted of a core of seven 90-min sessions administered over a 6-month period providing training in problem solving, communication skills, and specific education and behavioral management techniques in relation to caring for a child with TBI. These sessions included parents and school-aged children (children with TBI and siblings) and emphasized improving problem solving and communication for all members of the family. Parents of children in the treatment group reported significantly greater improvements in internalizing symptoms, anxiety/depression, and withdrawal on the Child Behavior Checklist than did parents in the standard care comparison group. Additionally, parents and children rated the program as extremely helpful and the problem-solving process as easy to use. All parents endorsed increased knowledge in program content areas, including understanding of their child's injury and awareness of strategies to improve attention and behavior, as well as improved parent-child relationships.

Several issues contribute to the difficulty in conducting psychosocial interventions following pediatric TBI. First, the problems confronting children following TBI and their families are multifaceted and vary substantially across families (Singer \& Powers, 1997; Wade et al., 1996). As a result, intervention models need to target multiple areas of vulnerability in a comprehensive intervention plan. Second, the family's needs are likely to change over the course of the child's recovery (Wade, Drotar, Taylor, \& Stancin, 1995). For example, during the acute hospitalization, the family's primary concern is the child's survival. For children receiving rehabilitation, the services are short-term and emphasize helping the child to function again at home and school. Only after inpatient rehabilitation is complete do families begin to confront how to manage persistent behavioral and cognitive changes in the child (Max, Castillo, et al., 1998). Family adaptation is an ongoing process influenced by the child's stage of recovery as well as normal family development (e.g., sibling rebellion, birth of a child) and outside events (e.g., loss of a job, illness). Thus, any intervention for pediatric TBI must take into account the child's stage of recovery, the family's level of development, and other life stresses confronting the family. In addition to commonly encountered obstacles to obtaining psychological care, such as arranging transportation and child care for other children and coordinating school and work schedules, families of children with TBI face several unique barriers. Pediatric trauma and rehabilitation services are centralized in urban, university-affiliated hospitals, with inpatients drawn from broad geographic areas spanning hundreds of miles. Thus, returning to the rehabilitation facility for ongoing counseling may be impossible. Additionally, there may be few, if any, other children with TBI in the child's home community, and local mental health providers and educators may lack the requisite knowledge and expertise for dealing with children who have TBI. Thus, families are forced to travel prohibitively long distances weekly for a period of months or years to obtain services or to forgo them altogether.

\section{Emerging Technologies and On-Line Therapeutic Interventions}

Computers have been used to facilitate mental health assessment and treatment for nearly 30 years (Greist et al., 1973), and advances in information technology associated with the Internet and World-Wide Web (WWW, or Web) ensure that e-health applications will continue to grow (Maheu, 2000). According to the 2000 census, $41.5 \%$ of households have Internet access. Moreover, mental health issues are the second most commonly searched health concern on the Internet (Rabasca, 2000). However, with some noteworthy exceptions, on-line interventions have been adopted infrequently with pediatric populations (see Farmer \& Muhlenbruck, 2001; Wade, 2004). Comparisons of on-line interventions with traditional face-toface therapy indicate that on-line treatment results in comparable outcomes while possessing several advantages over conventional treatment (Greist, 1998; I. Marks, Shaw, \& Parkin, 1998; Sturges, 1998). Accessibility is a key advantage since on-line services that do not involve a therapist can be accessed from home during nonworking hours. Additionally, clients can have more therapeutic time since the client can review on-line materials repeatedly without involving a therapist's time. Participants in on-line assessment and treatment also report less stigma and greater perceived confidentiality (Gega, Marks, \& Mataix-Cols, 2004). In fact, there is some evidence that individuals may prefer the neutrality of computerprovided services to face-to-face interaction with a therapist (Hewson, Laurent, \& Vogel, 1996; Joinson, 1998). Well-designed on-line treatment programs that incorporate multimedia or hypermedia presentations can facilitate education for individuals with learning disabilities or low literacy.

On-line or computer-mediated approaches have not been widely used with families, although existing stud- 
ies indicate considerable potential (Glueckauf et al., 2002; Ritterband et al., 2003). Glueckauf and colleagues reported that home-based family videoconferencing, phone conferencing, and face-to-face meetings were equally effective in reducing the frequency and severity of problem behaviors in teenagers with epilepsy. Similarly, Ritterband et al. found that an interactive on-line family intervention for encopresis was successful in significantly reducing the frequency of soiling. These limited findings suggest that on-line interventions can be effectively used with families as well as individuals.

Potential drawbacks of computer-assisted treatment, such as discomfort with technology and lack of personalization, can be addressed by providing face-to-face contact at the beginning of treatment (Maheu, 2003). Thus, computers have the potential to provide some benefit with a variety of conditions and may be especially helpful for problems where access to care is limited or where multimodal presentation is beneficial.

Families of children with TBI may be particularly able to benefit from an on-line intervention. Common barriers to treatment such as time, distance, and the unavailability of providers knowledgeable about TBI are eliminated when treatment is provided on line. Moreover, families can access treatment at any time during the course of recovery, returning to the on-line materials when new problems emerge. Although a number of Web sites currently provide information and referral resources regarding brain injury (e.g., the Brain Injury Association), most are geared toward brain injury in adults and none provides synchronous counseling with a knowledgeable provider.

\section{Innovation}

This report describes the development of and preliminary feasibility and usability data for an on-line treatment program, Family Problem Solving (FPS), adapted from a previously developed face-to-face intervention (Wade et al., 2004). FPS addresses cognitive appraisals, coping, and family communication through structured, self-guided Web pages and synchronous one-on-one videoconference sessions with a trained therapist. Allowing families to access help on line has the potential to significantly reduce both physical and psychological barriers to care. This project represents one of the first Web-based interventions designed to be used by a family and to solicit feedback from both individual family members and the family as a group; moreover, this intervention is perhaps the first that integrates self-guided Web materials with synchronized videoconferencing between the family and therapist. This study tests the following hypothesis: Can families of children with TBI that have varied prior computer experience successfully use and benefit from this Webbased intervention?

\section{METHOD}

\section{Participants}

Participants were recruited from the trauma registry of an urban children's hospital. To be eligible, children had to be between the ages of 5 and 16 and to have sustained a moderate to severe trau- matic brain injury more than 15 months previously. Consistent with other investigations, severe brain injury was defined as a lowest Glasgow Coma Scale (GCS) score of 8 or lower. Moderate TBI was defined as a GCS score of 9-12, or a higher GCS score with evidence of brain abnormalities on imaging studies (Fletcher et al., 1990). Children with nonblunt head trauma (e.g., gunshot wounds) were excluded.

A total of 19 participants in six families were recruited to participate in the FPS feasibility project. The 2 girls and 4 boys with TBI were an average of 9.4 years of age (range: 6 years 8 months to 15 years 9 months) and had been injured 16 months ago on average (range: 15-29 months). One child was African American and 1 was biracial. In four families (67\%), the parents were married and residing together in the home. SES and education level varied substantially. The occupation of participating parents included janitor, construction worker, and lawyer; one parent was unemployed. Parental education ranged from high school dropout to advanced degree. Four families had existing home computers (used only by children in one case) while the other two had very limited previous computer experience.

\section{Procedure}

After the research assistant recruited the participants and obtained informed consent, she went to the family's house and installed a Dell computer with 19-in. monitor, DeskJet printer, and Web camera. Families were allowed to keep the computers and printers at treatment completion. High-speed Internet access was also installed (when possible). We were able to obtain high-speed connections (four cable, one DSL) for all but one family. During this visit, the research assistant provided the family members with training in how to turn on the computer, access the Web site via Internet Explorer, and navigate within the Web site. With the exception of the family that was unable to receive high-speed Internet access, families were randomly assigned NetMeeting, an Orange Microsystems iBOT Web camera, and a microphone, or a Polycom ViaVideo Web camera that included an integrated microphone and videoconferencing software. Both platforms allowed two-way audio and video and application sharing; however, when used in two-way videoconferencing, they differed in picture size at $352 \times 288$ pixels (NetMeeting: $2 \times 2$ in., see Figure 1; and ViaVideo: $10 \times 8$ in., see Figure 2) and video- and sound-enhancing features (ViaVideo had several incorporated in the software). The platforms also differed substantially in cost $(\mathrm{ViaVideo}=\$ 400$ with discount; iBOT $=\$ 80)$. Because ViaVideo can only be used with a high-speed connection, the family with a dial-up connection received an iBOT. They also received a speakerphone rather than a microphone to minimize the demands on the limited bandwidth, and a second, dedicated, phone line paid for by the project. Part of the purpose of this feasibility trial was to determine whether the larger/higher quality video image would substantially change the family's experience with the intervention and would thus be worth the additional expense.

After the computers and Internet connections were installed, the therapist met once with each family in the family's home to conduct a structured interview/assessment of the family and to establish rapport. The latter portion of this visit was spent on the Web site reviewing the content and structure of the intervention and entering goals that each individual wanted to address during the intervention. Both of the parents, the injured child, and school-aged siblings were invited to participate in this meeting. Subsequent weekly meetings with the therapist were conducted via videoconferencing. Prior to each meeting, the family was asked to complete self-guided materials on the Web site. (A complete description of the intervention Web site is provided below.) At the sixth videoconference, the therapist and family members reviewed their progress and outstanding concerns. Up to four additional videoconferences could be scheduled depending on the stressors and skill deficits identified on the self-report measures completed at Session 6. A final video conference was scheduled for all families. 


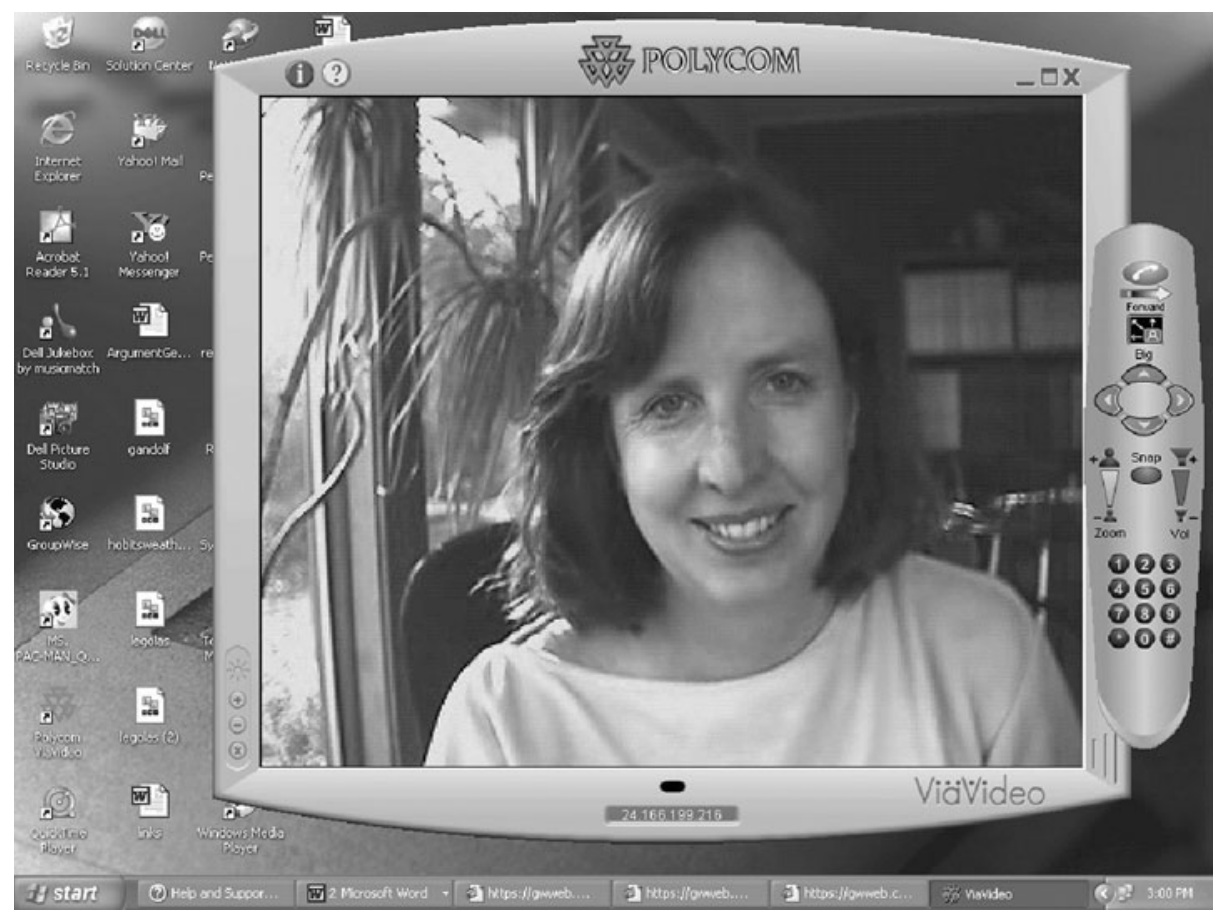

Figure 1. ViaVideo image on the desktop.

\section{Description of the FPS Intervention}

The FPS Web site was developed in accordance with Wolfe's (2001) principles of Web-based learning. The FPS homepage featured buttons that when clicked took participants to announcements, contact information, resources (other brain injury Web sites), and session materials. Under the Start Sessions button, participants were able to access Web site content that had been assigned to them, with new sessions being assigned by the therapist or coach after previous sessions had been completed. All families were assigned the same seven core on-line sessions in sequence.

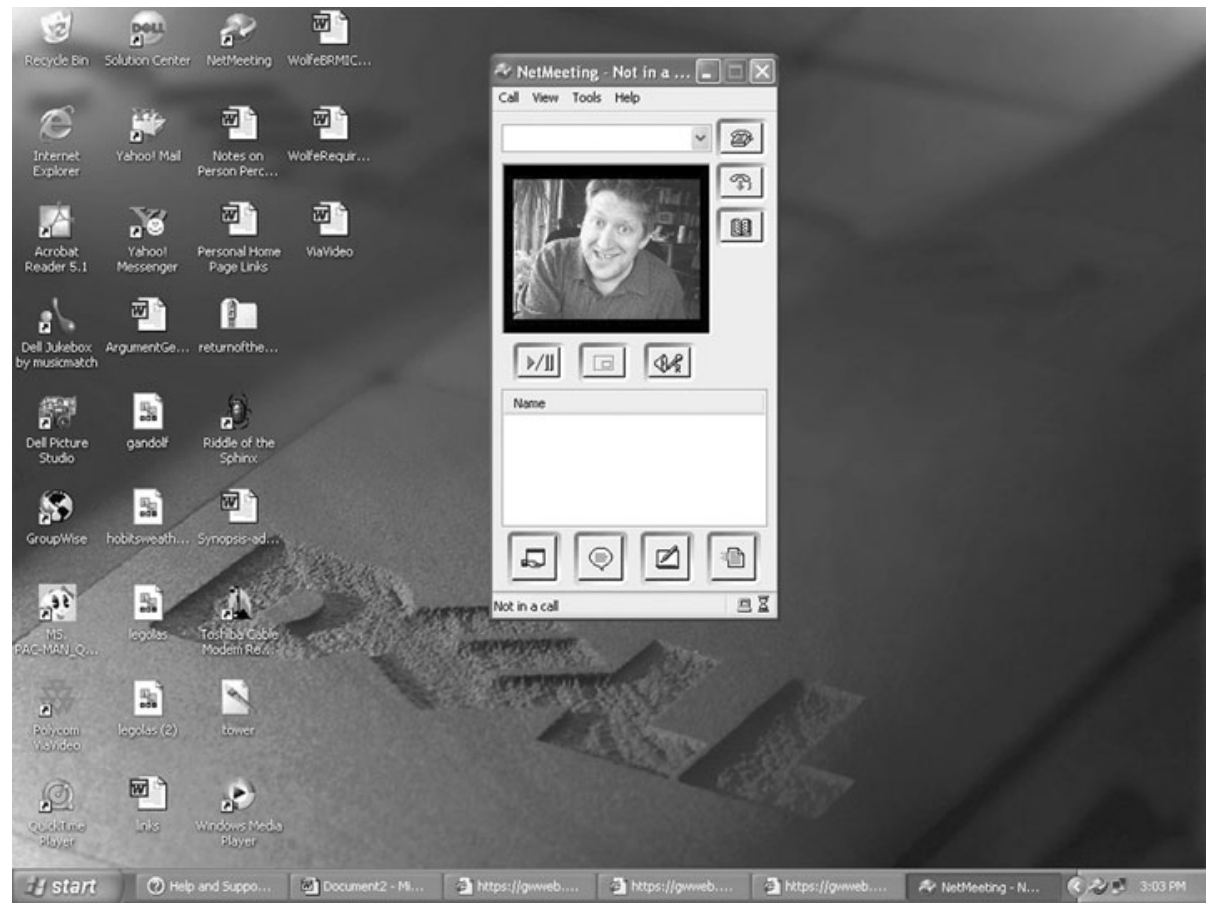

Figure 2. NetMeeting image on the desktop. 


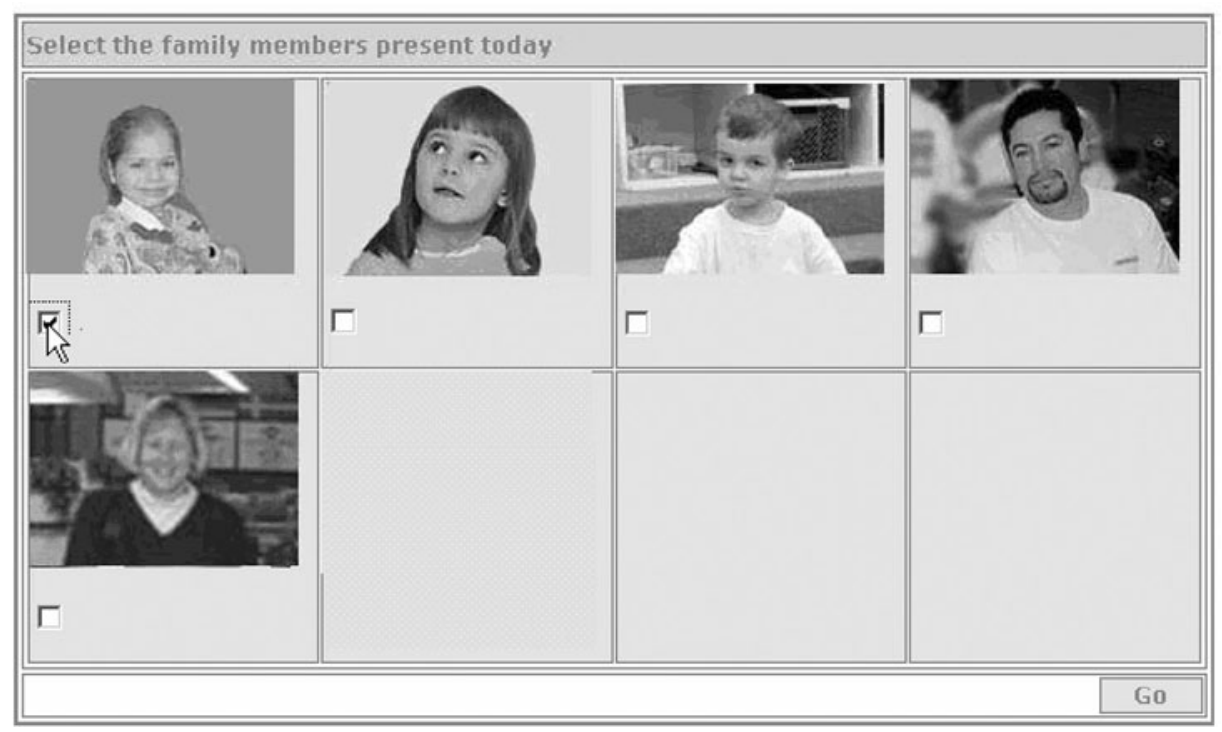

Figure 3. Log-on screen for participating family members.

Additional sessions were assigned on the basis of family needs. For example, families would be assigned the additional session "Working with the Schools" if that was an area of concern. A distinguishing characteristic of this Web site is that it was designed for family members to visit together. Family members logged in together by clicking under their picture to indicate that they were present (Figure 3). Some family members were required to be at each session, whereas the presence of others was optional for some sessions. Throughout the sessions, the system would sometimes prompt specific family members to respond in turn individually, while at other times a single response was required from the whole family.

The FPS Web site had 12 separate sessions. Eight core sessions provided problem solving, communication, and TBI-specific behavior management skills to all enrolled families, and the remaining four sessions addressed content related to the unique stressors and burdens of individual families. Self-guided on-line Web sessions included didactic content regarding the skill (e.g., problem solving), video clips showing individuals and families modeling the skill, and exercises and assignments giving the family an opportunity to practice the skill. Family members were asked to discuss certain problems or issues off-line before responding on the Web site. Families were also asked to respond, both as individuals and as a group, to questions, exercises, and problems by entering their responses to queries on the Web site. Photographs of individual family members would appear when responses from that individual were required. Participating families could access assigned materials at any time (day or night). Families received corrective feedback regarding on-line exercises and assignments through the Web pages. Additionally, exercises completed by the family were stored for review by the therapist prior to synchronous videoconference sessions with the family. This structure allowed us to provide a Web site that was both standardized and tailored to individual needs. The second part of each session involved a synchronous on-line appointment with the therapist to answer questions, review the exercises/ worksheets, and implement the problem-solving process with a problem identified by the family. These sessions were $45-60 \mathrm{~min}$ in length and involved all participating family members.

All data were stored and accessed in accordance with the HCFA's Internet Security Policy and the Health Insurance Portability and Accountability Act of 1996 (HIPAA), which provides guidelines for handling clinical data. The data collection systems incorporated a multilayered data security approach through the use of roles, user accounts, and passwords. Standard 128-bit encryption was done during transmission and authentication. Secure data were also protected by multiple, redundant layers of firewall systems.

\section{Measures}

Both qualitative and quantitative methods were used to assess the family's experience with the Web site, associated technology, and intervention content. First, parents, children, and participating siblings completed a survey assessing satisfaction with the intervention protocol and materials. Second, participants completed the Web Site Evaluation Questionnaire (WEQ), adapted from the Web site evaluation instrument created for an adult TBI project (Rotondi, Sinkule, \& Spring, in press). Third, parents completed a seven-item version of the Agnew Relationship Measure (ARM; Agnew-Davies, Stiles, Hardy, Barkham, \& Shapiro, 1998) to assess the quality of their relationship with the therapist. Finally, participants (both parents and participating children and siblings) were debriefed in qualitative interviews to solicit their perceptions of the strengths and weaknesses of the Web site and videoconferencing and to obtain suggestions for change.

\section{Analyses}

Frequency analyses and descriptive statistics were conducted on the quantitative outcomes. We used $t$ tests to compare WEQ ratings and satisfaction scores for individuals receiving the $\mathrm{BBOT}$ and ViaVideo Web cameras. Analysis of variance was used to compare WEQ ratings for parents, children with TBI, and siblings.

\section{RESULTS}

\section{Feasibility}

A total of 19 individuals participated in the study, including 6 children with TBI, 8 parents, and 5 siblings. Families completed 10.3 Web sessions on average (range: 7-12) and 10.1 synchronous videoconferences (range: $7-14)$. All Web sessions $(n=62)$ were completed independently by the families without therapist assistance, 
and this did not vary by prior computer ownership. The fact that all of the families were able to complete the intervention provides preliminary evidence that this approach is feasible for families with varied education and computer experience.

\section{Ease of Use}

Participating family members ranked the Web site as moderately to very easy to use overall $(M=3.59 / 5)$ and in comparison with other Web sites $(M=3.47 / 5 ; 3=$ moderately easy, $4=$ very easy; Table 1$)$. Logging on and navigating were rated as less difficult than completing questionnaires, although this difference was not statistically significant (logging on $M=3.82$; navigating $M=$ 4.00; questionnaires $M=3.53$ ). Ratings of Web site ease of use did not differ by camera type, age of respondent, or relationship to the child (self, sibling, or parent). The videoconferences were also rated moderately to very easy to use overall and when compared with a phone call or face-to-face visit (Table 1). However, family members with ViaVideo cameras rated videoconferencing significantly more favorably relative to face-to-face meetings (4.22/5) than did those with iBOT cameras (2.75/5). Although ratings of videoconference ease of use did not differ by age or relationship to the child, information from the qualitative interviews suggested that younger children and those with attention difficulties found the smaller video image provided by NetMeeting to be inadequate to sustain their attention during the videoconferencing. Moreover, all families using NetMeeting commented on the poor audio quality and nearly constant audio feedback and reverberation. Although 12/19 participants $(63 \%)$ preferred meeting on line to face-to-face, 1 sibling, 3 children with TBI, and 3 parents indicated that they would rather meet in person. Interestingly, all but one of these was using NetMeeting for videoconferences. Qualitative responses shed light on the reasons for preferring one mode of contact or another. Proponents of videoconferencing indicated perceived confidentiality and the convenience of accessing services within one's home. Those who reported a preference for meet-

Table 1

Ratings of Ease of Use of Web Site and Videoconferencing by Web Camera Item

\begin{tabular}{|c|c|c|c|c|c|c|}
\hline & \multicolumn{2}{|c|}{$\begin{array}{c}\text { ViaVideo } \\
\text { Rating } \\
(n=9) \\
\end{array}$} & \multicolumn{2}{|c|}{$\begin{array}{l}\text { iBOT } \\
\text { Rating } \\
(n=8) \\
\end{array}$} & \multicolumn{2}{|c|}{$\begin{array}{c}\text { Overall } \\
(n=17)\end{array}$} \\
\hline & $M$ & $S D$ & $M$ & $\overline{S D}$ & $M$ & $S D$ \\
\hline \multicolumn{7}{|l|}{ Web Site } \\
\hline Overall & 3.78 & .67 & 3.13 & 1.46 & 3.47 & 1.12 \\
\hline Logging on & 4.22 & .83 & 3.38 & 1.41 & 3.82 & 1.18 \\
\hline Navigating & 4.00 & 1.12 & 4.00 & 1.07 & 4.00 & 1.06 \\
\hline Questionnaires & 3.44 & 1.01 & 3.63 & 1.19 & 3.53 & 1.07 \\
\hline Versus other sites & 3.89 & .93 & 3.25 & .87 & 3.59 & .94 \\
\hline \multicolumn{7}{|l|}{ Videoconferencing } \\
\hline Overall & 4.22 & .67 & 3.25 & 1.39 & 3.76 & 1.15 \\
\hline Versus face-to-face* & 4.22 & .83 & 2.75 & 1.58 & 3.53 & 1.42 \\
\hline Versus a phone call & 4.22 & .83 & 3.00 & 1.51 & 3.65 & 1.32 \\
\hline
\end{tabular}

Note-Ratings of helpfulness: $5=$ extremely/completely $; 4=$ very $; 3=$ moderately; $2=$ a little $1=$ not at all. ${ }^{*}$ Group difference $p<.05$. ing face-to-face noted the difficulty of listening and sustaining attention to the computer.

\section{Ratings of Helpfulness and Treatment Satisfaction}

Perceptions of the helpfulness of the intervention were high, with both the Web site and videoconferencing rated very helpful overall $(M=4.12$ and 4.35 , respectively; $4=$ very; 5 = extremely; see Table 2 ). Of the specific components of the Web site, the information on brain injury was rated most highly $(M=4.65 / 5)$. Qualitative feedback regarding the brain injury content was also very positive. In addition to increased understanding of TBI, parents reported that the information gave them a sense of closure and helped them to feel less alone. The other components were rated as very helpful as well $(M=3.96 / 5)$. Parents and older children described the problem-solving process as "really useful" and applicable to future problems. Although the effect was not statistically significant, families using NetMeeting rated the videoconferences as less helpful relative to a face-toface visit or phone call than did those using ViaVideo (mean face-to-face 3.00 vs. 4.44 ). Satisfaction with the intervention was also high (Table 3). All parents and siblings and all but 1 child with TBI (94.7\% of total) indicated that they would recommend the program to others. Parents, children with TBI, and siblings all rated the program as very helpful $(M>9 / 10)$. Again, the convenience of meeting within one's home was noted as a factor in perceptions of satisfaction. Parents rated the program as more enjoyable than did the child with TBI or his/her siblings. Children found the information more difficult to understand, and, as noted above, the varied audio and visual quality of the videoconferences interfered with their attention and comprehension.

\section{Reported Improvements in Target Behaviors}

Parent and child ratings suggest that the program resulted in improved outcomes on target behaviors for all families (Table 4). All parents indicated that they understood their child and his/her injury better, got along with their child better, and had a plan for handling future problems. Children with TBI also reported increased understanding of their injury ( $83.3 \%$ ), awareness of strategies to improve attention (100\%), and improved relationships with their parents $(100 \%)$. Siblings also reported understanding their sibling and his/her injury better $(100 \%)$, getting along with their parents better $(100 \%)$, getting along with their siblings better $(80 \%)$, and feeling less stressed $(80 \%)$.

\section{Therapeutic Alliance}

Despite the remote nature of the treatment, parents reported a strong therapeutic alliance, as indicated by high levels of confidence in the therapist and her skills $(M=$ $6.75 ; 6.88$ on a 7 -point scale), comfort in openly expressing oneself $(M=6.38)$, and agreement on how to work together $(M=6.14)$. The children with TBI and siblings also rated the therapist as caring $(M=9.67$ and 
Table 2

Ratings of Helpfulness of Specific Intervention Content/Components by Web Camera Item

\begin{tabular}{|c|c|c|c|c|c|c|}
\hline & \multicolumn{2}{|c|}{$\begin{array}{c}\text { ViaVideo } \\
\text { Rating } \\
(n=9)\end{array}$} & \multicolumn{2}{|c|}{$\begin{array}{l}\text { iBOT } \\
\text { Rating } \\
(n=8)\end{array}$} & \multicolumn{2}{|c|}{$\begin{array}{c}\text { Overall } \\
(n=17)\end{array}$} \\
\hline & $M$ & $S D$ & $M$ & $S D$ & $M$ & $S D$ \\
\hline \multicolumn{7}{|l|}{ Web Site } \\
\hline Overall & 4.11 & 1.05 & 4.13 & .35 & 4.12 & .78 \\
\hline TBI information & 4.78 & .44 & 4.50 & .76 & 4.65 & .61 \\
\hline Problem solving & 4.00 & 1.00 & 3.88 & .35 & 3.94 & .75 \\
\hline Behavior strategies & 4.00 & .87 & 3.88 & .35 & 3.94 & .75 \\
\hline Communication skills & 4.22 & .67 & 3.75 & .71 & 4.00 & .71 \\
\hline \multicolumn{7}{|l|}{ Videoconferencing } \\
\hline Overall & 4.44 & .73 & 4.35 & 1.03 & 4.35 & .86 \\
\hline Versus face-to-face* & 4.44 & .73 & 3.00 & 1.78 & 3.76 & 1.48 \\
\hline Versus a phone call & 4.33 & .87 & 3.38 & 1.19 & 3.88 & 1.11 \\
\hline
\end{tabular}

Note-Ratings of helpfulness: $5=$ extremely/completely; $4=$ very; $3=$ moderately $; 2=$ a little $; 1=$ not at all. ${ }^{*}$ Group difference approaches significance, $p=.06$.

8.40 on a 10 -point scale, respectively). During the qualitative interviews, parents and children described the experience with FPS as more helpful than previous experiences with psychotherapy and TBI specialists, noting the positive, skill-building emphasis of the intervention and the frequent feedback from the therapist. Parents also indicated that the program provided more specifics for coping with the sequelae of brain injury than had previous professionals: "You didn't just diagnose the problem, you told us how to deal with it." The children with TBI also rated the program positively compared with other therapy because it was more "relaxed" and didn't involve long car trips.

\section{The Therapist's Experience}

The therapist, a PhD-level pediatric psychologist, had no experience with videoconferencing prior to this project. The time the therapist spent preparing and conducting videoconference therapy sessions with participating families was comparable to the amount of time she would have devoted during conventional therapy. However, troubleshooting around intermittent technical problems added to the time spent with each family. Additionally, approximately $20 \%$ of sessions had to be rescheduled due to problems accessing the Web site (see below) or difficulties establishing an acceptable video connection. The therapist's comfort with the technology and the mode of treatment delivery increased substantially over the

Table 3

Ratings of Satisfaction with the Intervention

\begin{tabular}{|c|c|c|c|c|c|c|}
\hline \multirow[b]{2}{*}{ Item } & \multicolumn{2}{|c|}{$\begin{array}{c}\text { Parent } \\
(n=8)\end{array}$} & \multicolumn{2}{|c|}{$\begin{array}{l}\text { Child } \\
(n=6)\end{array}$} & \multicolumn{2}{|c|}{$\begin{array}{l}\text { Sibling } \\
(n=5)\end{array}$} \\
\hline & $M$ & $S D$ & $M$ & $S D$ & $M$ & $S D$ \\
\hline Overall helpfulness & 9.25 & .89 & 9.50 & .55 & 9.00 & 2.24 \\
\hline Therapist & 9.88 & .35 & 9.67 & .52 & 8.40 & 2.07 \\
\hline Clarity of materials & 8.00 & 1.60 & 7.17 & 2.14 & 7.40 & 2.79 \\
\hline Information & 8.75 & .89 & 9.50 & .55 & 8.20 & 2.17 \\
\hline Enjoyed the program & 9.00 & .58 & 7.50 & 3.51 & 7.60 & 2.07 \\
\hline
\end{tabular}

Note-1 = not at all $; 10=$ extremely/completely. course of the intervention, and she reported feeling very comfortable with the technology and videoconferencing format by the end. However, the therapist noted a number of concerns. Specifically, she had difficulty hearing and seeing all participating family members at times, particularly with NetMeeting, which necessitated some repeated questioning. Initially, the therapist also reported feeling compelled to "project" her emotions and reactions like a stage actor to be understood by the family.

\section{Technical Difficulties}

Examination of feasibility would not be complete without consideration of the technical difficulties encountered. As noted above, audio quality through the computer was unacceptable with NetMeeting. Depending on time of day, audio quality with ViaVideo could be choppy and intermittent. In addition to these inherent limitations of integrated audio, half of the computers were infected with the blaster worm and sobig virus. Moreover, changes in the hospital firewall following these viruses resulted in all participants being kicked off the Web site after having been connected for only a few minutes at a time. Although this problem was corrected after a few weeks, most families had difficulty completing the self-guided Web pages during this period. Thus, family ratings of ease of use and satisfaction need to be interpreted with these difficulties in mind.

\section{DISCUSSION}

Our findings suggest that families of children with TBI with varied prior computer experience can successfully use a face-to-face treatment adapted for the WorldWide Web to acquire knowledge and skills. Families' ability to independently complete the self-guided sessions, coupled with relatively high ratings of ease of use, support the notion that a Web-based approach is feasible for parents of children with TBI.

However, these preliminary results also appear to indicate that characteristics of the conferencing software and of the individual participants influenced perceptions of ease of use, and, to a lesser degree, helpfulness. Specifically, families employing NetMeeting with an iBOT camera for their videoconferencing platform reported lower ease of use due to inferior audio and video quality. Additionally, younger children and those with brain injury expressed a preference for meeting face-to-face because it was harder to attend to and comprehend the therapist over the computer. Moreover, due to limitations in audio and visual input, the therapist was less able to perceive the child's lack of understanding and respond appropriately. Although these findings should be considered as tentative given the small and heterogeneous sample, they have potential implications for subsequent work in this area. First, the larger images and clearer audio afforded by more expensive Web cameras and video conferencing software appear to make a difference in the family's experience and are therefore arguably worth the additional expense. Second, implementing Web-based therapy with 
Table 4

Proportion of Parents and Children Reporting Improvements in Target Behaviors

\begin{tabular}{lcc}
\hline \multicolumn{1}{c}{ Target Behavior } & $\begin{array}{c}\text { Parent } \\
(n=8)\end{array}$ & $\begin{array}{c}\text { Child } \\
(n=6)\end{array}$ \\
\hline Understand injury better & $100 \%$ & $83.3 \%$ \\
Know strategies for attention/memory & $87.5 \%$ & $100 \%$ \\
Plan for future problems & $100 \%$ & $83.3 \%$ \\
Feel less stressed & $87.5 \%$ & $66.7 \%$ \\
Get along with child/parent better & $100 \%$ & $100 \%$ \\
Get along with spouse/siblings better & $75 \%$ & $66.7 \%$ \\
Doing better in school & - & $50 \%$ \\
\hline
\end{tabular}

very young or cognitively impaired children may require additional sensitivity to the child's level of awareness and comfort. These findings are consistent with those of Glueckauf and colleagues (2002), who reported that adolescents with epilepsy were less comfortable with videoconferencing than with face-to-face therapy, perhaps due to the cognitive limitations associated with their disorder. Given the fact that all of the children with TBI reported experiencing improvements despite these difficulties, the approach still appears feasible with these caveats in mind.

Our findings indicate that Web-based FPS is helpful as well as feasible. All participants reported improvements on one or more of the dimensions of functioning targeted by the intervention, including increased understanding of brain injury, reduced stress, and improved relationships. These results are very comparable to those from the previous face-to-face trial of family problem solving with families of children with TBI (Wade et al., 2004), suggesting that an on-line approach can be as successful as one delivered in person. These results are consistent with those of the other study that has implemented a family-centered intervention over the Web (Ritterband et al., 2003). It is noteworthy that virtually all participants with prior psychotherapy experience in the present project reported that FPS was more helpful than prior experiences with psychotherapy. Other investigators (see Schopp, Johnstone, \& Merrell, 2000) have also found that telehealth clients report more positive experiences than their face-to-face counterparts. Although the accessibility of on-line FPS likely contributed to this preference, participants also noted the structured nature of the intervention and its emphasis on building skills and solving problems.

Like Glueckauf et al. (2002), we found that participants were able to build a strong relationship with the therapist, despite the fact that face-to-face contact was limited to an initial session. In fact, participants described the FPS therapist as more caring and involved than previous professionals despite the remote nature of the contact. However, during the qualitative interviews, most participants indicated that the initial face-to-face interview was critical for establishing a sense of comfort and trust. This preference for some face-to-face contact is consistent with Maheu's $(2000,2003)$ recommendation that the initial assessment be conducted in person. Further research is necessary to determine the impor- tance of some direct contact in establishing a therapeutic alliance.

Participants' relatively favorable perceptions of ease of use and helpfulness must be considered in the context of the technical difficulties they encountered. Owing to difficulties with remote connections, all families had to learn to find their IP address and to initiate videoconferences with the therapist. Because of virus and firewall difficulties, families also experienced difficulties accessing the Web site at times. Because the site cycled through family members, requiring each individual to respond, if the Web site crashed while the family was completing a questionnaire, all family members had to complete their responses again. Given these frustrating difficulties, the Web site ratings must be viewed as a lower limit of the actual ease of use.

Previous researchers (Kleiner, Akers, Burke, \& Werner, 2002; Schopp et al., 2000) have reported that clinicians are less comfortable with incorporating new technology into their practice than are the consumers of health services. Likewise, the therapist in this study noted the awkwardness of not being able to clearly see and hear all participants at all times. Moreover, although the data indicated that technical difficulties did not compromise the therapeutic alliance, the therapist was not always able to gauge the impact of technology on the therapeutic relationship during the course of treatment. Thus, therapists may require technical support and peer encouragement to successfully adopt telehealth approaches in their practices.

These are preliminary findings, and care must be taken in generalizing from these results. The sample size was small, and we lacked a control group. Thus, failure to find age differences in ratings of ease of use by age may be an artifact of the small sample. It is also not possible to determine whether ratings of improved knowledge and relationships were attributable to factors other than the treatment (e.g., the desire to please the researchers).

These caveats notwithstanding, our results underscore the potential viability and utility of Web-based approaches for families of children with TBI. The findings also point to crucial directions for improving on-line FPS, including ensuring a high-quality audio and visual connection and making the materials more child friendly. Future research is necessary to determine whether visual contact is necessary or whether phone contact with synchronous application sharing would be adequate. Randomized clinical trials are necessary to examine the efficacy of on-line FPS versus standard care. It would also be very useful to compare on-line FPS with access to on-line information. Nonetheless, the results provide important initial evidence of the feasibility of family-centered online interventions with stressed individuals drawn from all walks of life.

\section{REFERENCES}

Agnew-Davies, R., Stiles, W. B., Hardy, G. E., Barkham, M., \& ShapIRo, D. A. (1998). Alliance structure assessed by the Agnew Relationship Measure (ARM). British Journal of Clinical Psychology, 37, 155-172. 
BRooks, D. (1991). The head-injury family. Journal of Clinical \& Experimental Neuropsychology, 13, 155-188.

Brown, G., Chadwick, O., Shaffer, D., Rutter, M., \& Traub, M. (1981). A prospective study of children with head injuries: III. Psychiatric sequelae. Psychological Medicine, 11, 63-78.

Farmer, J. E., \& MuhlenbrucK, L. (2001). Telehealth for children with special health care needs: Promoting comprehensive systems of care. Clinical Pediatrics, 40, 93-98.

Fay, G. C., Jaffe, K. M., Polissar, N. L., Liao, S., Rivara, J. B., \& Martin, K. M. (1994). Outcome of pediatric traumatic brain injury at three years: A cohort study. Archives of Physical Medicine \& Rehabilitation, 75, 733-741.

Fletcher, J., Ewing-Cobbs, L., Miner, M., Levin, H., \& Eisenberg, H. (1990). Behavioral changes after closed head injury in children. Journal of Consulting \& Clinical Psychology, 58, 93-98.

Gega, L., Marks, I., \& MataIX-Cols, D. (2004). Computer-aided CBT self-help for anxiety and depressive disorders: Experience of a London clinic and future directions. Journal of Clinical Psychology, 60, 147-157.

Glueckauf, R. L., Fritz, S. P., EcKlund-Johnson, E. P., Liss, H. J., Dages, P., \& CARNEY, P. (2002). Videoconferencing-based family counseling for rural teenagers with epilepsy: Phase 1 findings. Rehabilitation Psychology, 47, 49-72.

GreIST, J. H. (1998). Treatment for all: The computer as patient assistant. Psychiatric Services, 49, 887-889.

Greist, J. H., Gustafson, D. H., Stauss, F. F., Rowse, G. L., LaughREN, T. P., \& CHILES, J. A. (1973). Computer interview for suiciderisk prediction. American Journal of Psychiatry, 130, 1327-1332.

Hawley, C. A., Ward, A. B., Magnay, A. R., \& Long, J. (2002). Children's brain injury: A postal follow-up of 525 children from one health region in the UK. Brain Injury, 16, 969-985.

Hewson, C. M., Laurent, D., \& Vogel, C. M. (1996). Proper methodologies for psychological and sociological studies conducted via the Internet. Behavior Research Methods, Instruments, \& Computers, 28, 186-191.

JoInson, A. N. (1998). Causes and implications of disinhibited behavior on the Net. In J. Gackenbach (Ed.), Psychology of the Internet (pp. 43-60). New York: Academic Press.

Kinsella, G., Ong, B., Murtaugh, D., Prior, M., \& Sawyer, M. (1999). The role of the family for behavioral outcome in children and adolescents following traumatic brain injury. Journal of Consulting \& Clinical Psychology, 67, 116-123.

Kleiner, K. D., Akers, R., Burke, B. L., \& Werner, E. J. (2002). Parent and physician attitudes regarding electronic communication in pediatric practices. Pediatrics, 109, 740-744.

Maheu, M. M. (2000). Delivering behavioral telehealth via the Internet: E-health. TelehealthNet. http://telehealth.net/articles/.

Maheu, M. M. (2003). The online clinical practice management model. Psychotherapy: Theory, Research, Practice, Training, 40, 20-32.

Marks, I., ShaW, S., \& PARKIN, R. (1998). Computer-aided treatments of mental health problems. Clinical Psychology: Science \& Practice, $\mathbf{5}, 151-170$

MARKs, M., Sliwinski, M., \& GoRdon, W. A. (1993). An examination of the needs of families with a brain injured child. NeuroRehabilitation, 3, 1-12.

Max, J. E., Castillo, C. S., Robin, D. A., Lindgren, S. D., Smith, W. L., JR., Sato, Y., Mattheis, P. J., \& Stierwalt, J. A. G. (1998). Predictors of family functioning after traumatic brain injury in children and adolescents. Journal of the American Academy of Child \& Adolescent Psychiatry, 37, 83-90.

MaX, J. E., Koele, S. L., Smith, W. L., Jr., Sato, Y., Lindgren, S. D., Robin, D. A., \& ARNDT, S. (1998). Psychiatric disorders in children and adolescents after severe traumatic brain injury: A controlled study. Journal of the American Academy of Child \& Adolescent Psychiatry, 37, 832-840.

RabascA, L. (2000). Self-help sites: A blessing or a bane? Monitor on Psychology, 31, 28-30.

Ritterband, L. M., Cox, D. J., Walker, L. S., Kovatchev, B., McKnight, L., Patel, K., Borowitz, S., \& SutPhen, J. (2003). An Internet intervention as adjunctive therapy for pediatric encopresis. Journal of Consulting \& Clinical Psychology, 71, 910-917.

Rivara, J. B., Fay, G. C., Jaffe, K. M., Polissar, N. L., Shurtleff,
H. A., \& Martin, K. M. (1992). Predictors of family functioning one year following traumatic brain injury in children. Archives of Physical Medicine \& Rehabilitation, 73, 899-910.

Rivara, J. B., Jaffe, K. M., Polissar, N. L., Fay, G. C., Liao, S., \& Martin, K. M. (1996). Predictors of family functioning and change 3 years after traumatic brain injury in children. Archives of Physical Medicine \& Rehabilitation, 77, 754-764.

Rotondi, A. J., Sinkule, J., \& Spring, M. (in press). An interactive Web-based intervention for persons with TBI and their families: Use and evaluation by female significant others. Journal of Head Trauma Rehabilitation.

Rutter, M. (1981). Psychological sequelae of brain damage in children. American Journal of Psychiatry, 138, 1533-1543.

Schopp, L. H., Johnstone, B. J., \& Merrell, D. (2000). Telehealth and neuropsychological assessment: New opportunities for psychologists. Professional Psychology: Research \& Practice, 31, 179-183.

Schwartz, L., Taylor, H. G., Drotar, D., Yeates, K. O., Wade, S. L., \& STANCIN, T. (2003). Long-term behavior problems following pediatric traumatic brain injury: Prevalence, predictors, and correlates. Journal of Pediatric Psychology, 28, 251-263.

Singer, G. H. S., Glang, A., Nixon, C., Cooley, E., Kerns, K. A., Williams, D., \& Powers, L. E. (1994). A comparison of two psychosocial interventions for parents of children with acquired brain injury: An exploratory study. Journal of Head Trauma Rehabilitation, 9, 3849.

Singer, G. H. S., \& Powers, L. E. (1997). Stress management training to help parents adapt to a child's ABI. In G. H. S. Singer, A. Glang, \& J. M. Williams (Eds.), Children with acquired brain injury: Educating and supporting families (pp. 235-251). Baltimore: Brookes.

Sturges, J. W. (1998). Practical use of technology in professional practice. Professional Psychology: Research \& Practice, 29, 183-188.

Taylor, H. G., Yeates, K. O., Wade, S. L., Drotar, D., Klein, S., \& STANCIN, T. (1999). Influences on first-year recovery from traumatic brain injury in children. Neuropsychology, 13, 76-89.

Taylor, H. G., Yeates, K. O., Wade, S. L., Drotar, D., Stancin, T., \& Minich, N. (2002). A prospective study of short- and long-term outcomes after traumatic brain injury in children: Behavior and achievement. Neuropsychology, 16, 15-27.

WADE, S. L. (2004). Commentary: Computer-based interventions in pediatric psychology. Journal of Pediatric Psychology, 29, 269-272.

Wade, S. L., Drotar, D., Taylor, H. G., \& Stancin, T. (1995). Assessing the effects of traumatic brain injury (TBI) on family functioning: Conceptual and methodological issues. Journal of Pediatric Psychology, 20, 737-752.

Wade, S. L., Michaud, L., \& Brown, T. M. (2004). A family intervention to improve child outcomes following traumatic brain injury (TBI). Manuscript submitted for publication.

Wade, S. L., Taylor, H. G., Drotar, D., Stancin, T., \& Yeates, K. O. (1996). Childhood traumatic brain injury: Initial impact on the family. Journal of Learning Disabilities, 29, 652-661.

Wade, S. L., Taylor, H. G., Drotar, D., Stancin, T., \& Yeates, K. O. (1998). Family burden and adaptation following traumatic brain injury (TBI) in children. Pediatrics, 102, 110-116.

Wade, S. L., Taylor, H. G., Drotar, D., Stancin, T., Yeates, K. O., \& MinICH, N. M. (2002). A prospective study of long-term caregiver and family adaptation following brain injury in children. Journal of Head Trauma Rehabilitation, 17, 96-111.

Wolfe, C. R. (2001). Learning and teaching on the World Wide Web. San Diego: Academic Press.

Yeates, K. O., Taylor, H. G., Drotar, D., Wade, S. L. Klein, S., Stancin, T., \& Schatschneider, C. (1997). Pre-injury family environment as a determinant of recovery from traumatic brain injuries in school-age children. Journal of the International Neuropsychological Society, 3, 617-630.

Yeates, K. O., Taylor, H. G., Wade, S. L., Drotar, D., Stancin, T., $\&$ MinICH, N. (2002). A prospective study of short- and long-term neuropsychological outcomes after traumatic brain injury in children. Neuropsychology, 16, 514-523.

(Manuscript received December 12, 2003; revision accepted for publication April 5, 2004.) 